\title{
CdSe/ZnS core-shell nanocrystal based scintillators for enhanced detection in UV
}

\author{
Hilmi Volkan Demir, Ibrahim Murat Soganci, Evren Mutlugun \\ Department of Physics and Department of Electrical and Electronics Engineering, Nanotechnology Research Center, Bilkent University, \\ Ankara,06800 Turkey.Tel:[+90](312)290-1021 E-mail: volkan@bilkent.edu.tr
}

Nanocrystals that have radii smaller than the bulk exciton Bohr radius of the material exhibit discrete, atomic like energy levels due to the quantum confinement. These nanocrystals, also called as artificial atoms, exhibit ultranarrow optical transitions [1]. For nanocrystals with $\mathrm{CdSe}$ as the core and $\mathrm{ZnS}$ as the surrounding shell, the quantum efficiency of the fluorescence at room temperature may increase up to $50 \%$ across the visible spectral range [2]. Also, the absorption coefficient of such nanocrystals is very high at short wavelengths, covering the ultraviolet spectral range [3]. In this work, we present a novel scintillator that incorporates $\mathrm{CdSe} / \mathrm{ZnS}$ core-shell nanocrystals to utilize both their high quantum fluorescence efficiency in the visible and high optical absorption in the UV for the purpose of facilitating UV imaging on a Si platform. Here we demonstrate enhancement in UV detection up to $800 \%$ with respect to the host polymer in the UV.

The working principle of this scintillator relies on the absorption and emission spectra of the nanocrystals that are separated from each other. As a result of the high absorption coefficient in the UV region, incident ultraviolet light creates electron-hole pairs with high efficiency, and the resulting recombination occurs at the emission wavelength in the visible range, which is longer than the absorption wavelength. Thus, integrating nanocrystals, for example, on a Si platform, it is possible to have otherwise undetectable UV photons to be converted to the visible photons to be detected by the Si. Furthemore, the emission wavelength of the nanocrystals can be tuned to any desired point in the visible by simply changing the size of the nanocrystals as necessitated by the specifications of the scintillator. For more efficient device performance, the absorption and emission spectra of the nanocrystals need to have minimum overlap to avoid further absorption of the emitted light, which acts as a loss term for the scintillator. In Fig. 1a, the emission of a CdSe/ZnS nanocrystal film is presented. Here its excitation wavelength is $300 \mathrm{~nm}$ and its emission wavelength is $620 \mathrm{~nm}$. The absorption and photoluminescence spectra of this nanocrystal are also shown in Fig. 1b.

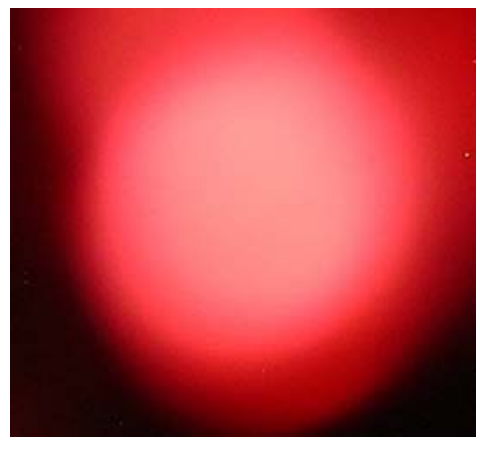

(a)

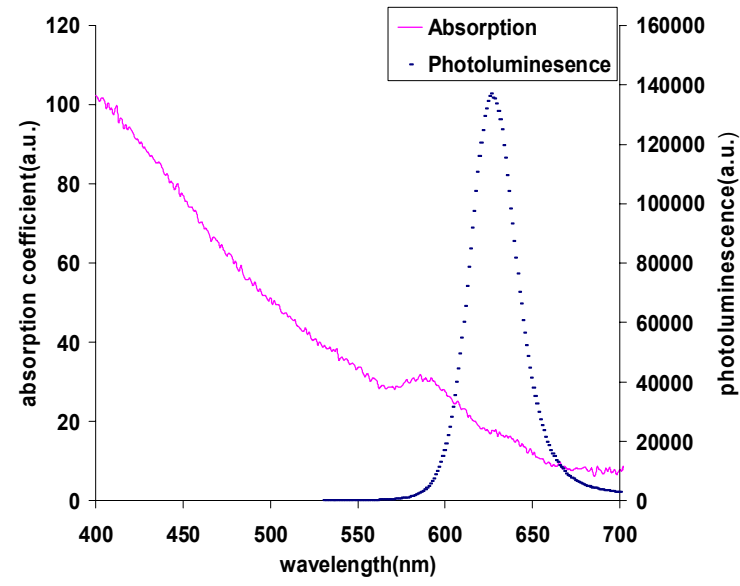

(b)

Figure 1:(a) Image of the emission of the red nanocrystals and (b) their emission and absorption spectra.

The excitation spectrum of the nanocrystals defines the efficiency of the scintillator, which is shown in Fig. 2a as a function of the excitation wavelength. This graph shows that the excitation efficiency is higher around $230 \mathrm{~nm}$. This means that the highest optical power at $620 \mathrm{~nm}$ is obtained when the nanocrystals are excited around that particular region of the ultraviolet range.

From the device point of view, the integration of the nanocrystals onto an optoelectronic device plays a great role for its future aspects. Figure $2 \mathrm{~b}$ shows the enhancement obtained in the detected power from the $\mathrm{Si}$ photodetector by using the nanocrystal coated quartz on top of the photodetector. Although the nanocrystals are in a host polymer that is highly absorbing in the UV region, the overall enhancement obtained is very promising. The 
nanocrystals exhibit great improvement (over \%800) in detected power with respect to their host polymer (Fig. 3a), which hold great promise for integrated device applications. Figure $3 \mathrm{~b}$ plots the simulated scintillator operation as a function of different quantum efficiencies along with the experimental measurement.

Similarly, we measure the performance of the nanocrystals in scintillator application by integrating an unpackaged silicon photodetector with $\mathrm{CdSe} / \mathrm{ZnS}$ nanocrystals onto the active region of the photodetector. The responsivity of the photodetector integrated with nanocrystals in the polymer matrix is measured to be more than 3.5 times higher than that of the photodetector with the sole polymer addition for the near ultraviolet region.

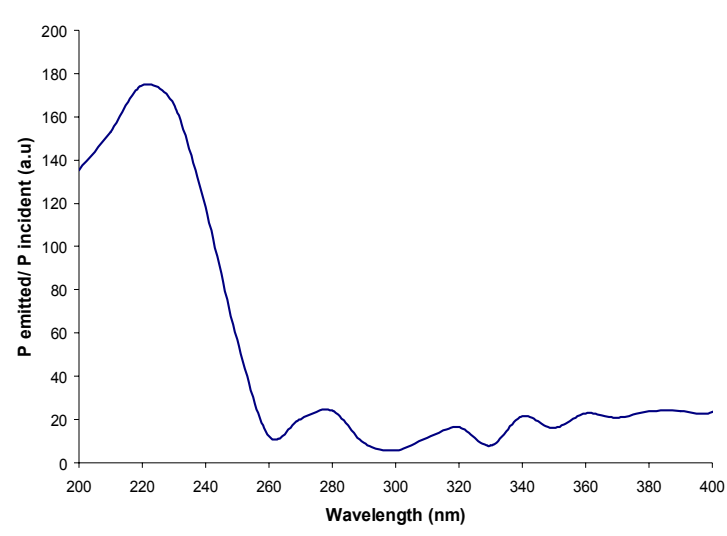

(a)

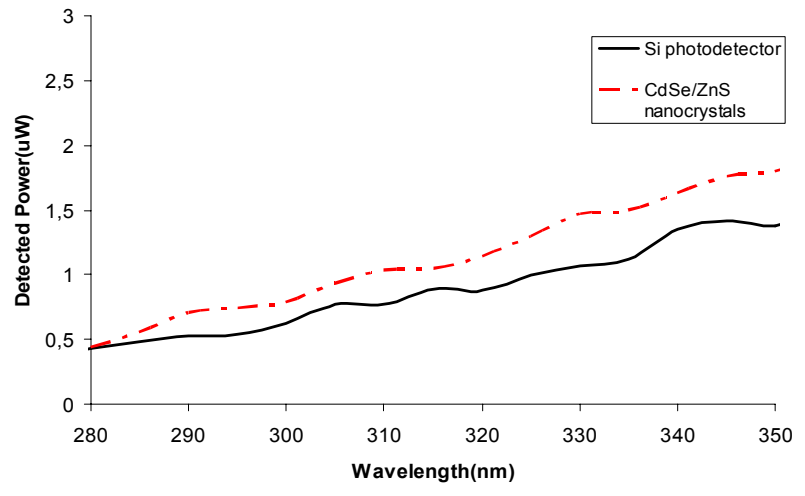

(b)

Figure 2:(a) Excitation spectra of the red nanocrystals and (b) the improvement in the detected power for the red nanocrystals with respect to the Si photodetector.

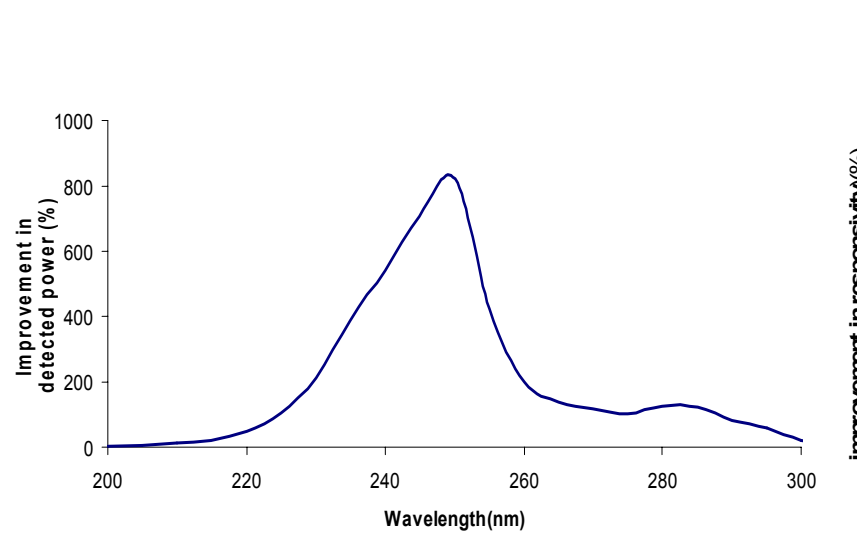

(a)

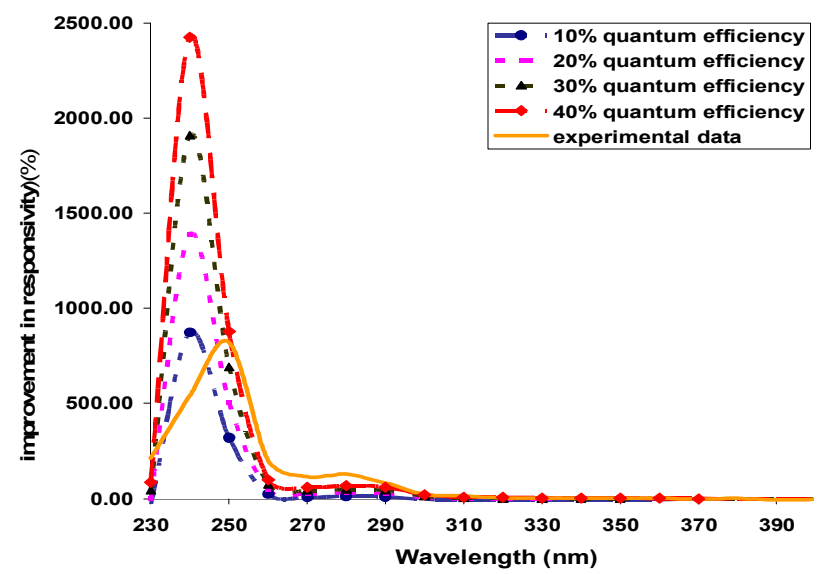

(b)

Figure 3: (a) The improvement in detected power for nanocrystals with respect to its host polymer (b) the simulation for different quantum efficiencies of CdSe-ZnS nanocrystals along with the experimental data.

In conclusion, we report the proof-of-concept demonstration of a scintillator that integrates $\mathrm{CdSe} / \mathrm{ZnS}$ core-shell nanocrystals for its functional use in enhanced UV detection.

Acknowledgements: This work is supported by EU MOON, EU NOE PHOREMOST and TUBITAK 104E114, 106E020, 105E065, and 105E066. H.V.D and I.M.S. also acknowledge partial support from Turkish Academy of Sciences and TUBITAK, respectively.

\section{References}

[1] S. A. Empedocles et al., Phys. Rev. Lett., 77, 18, (1996).

[2] B. A. Dabbousi, J. Phys. Chem. B, 101, 9463, (1997).

[3] C. A. Leatherdale et al., J. Phys. Chem. B, 106, 7619, (2002). 